\title{
The relationship between physical activity levels and cardiovascular disease risk factors in middle-aged women
}

\author{
Yuliang Sun ${ }^{1, a}$ \\ ${ }^{1}$ School of Physical Education, Shaanxi Normal University, Shaanxi 710119, China; \\ asunsyl123@163.com
}

Keywords: physical activity, cardiorespiratory fitness, cardiovascular disease risk

\begin{abstract}
High levels of physical activity (PA) and cardiorespiratory fitness (CRF) are each associated with a favorable cardiovascular disease (CVD) risk profile. The study included 231 urban-dwelling asymptomatic 40-49 year old women. Body mass index (BMI), body fat percentage (BF\%), blood glucose, blood lipids, blood pressure, and pulse wave velocity (PWV) were measured at rest. Cycle ergometer exercise tests were conducted to assess CRF as indicated by maximal oxygen uptake $\left(\mathrm{VO}_{2 \max }\right)$. Participants were categorized into three CRF levels (low, moderate and high). High CRF level was associated with significantly less BF\%, lower PWV, and higher weekly physical activity compared with low and moderate CRF $(\mathrm{P}<0.05)$. Compared to high CRF, the odds ratios for having $\geqslant 3$ main CVD risk factors (overweight, hypertension, and dyslipidemia) in low and moderate CRF were 2.09 (95\% CI: 1.48-2.94) and 1.84 (95\% CI: 1.29-2.62), respectively. Chinese middle-aged women demonstrated a moderate level of CRF. CRF was independently associated with CVD risk factors, including overweight, hypertension, dyslipidemia, arterial stiffness, and abnormal ECG during exercise, with the least fit women exhibiting the highest number of CVD risk factors.
\end{abstract}

\section{Introduction}

With China's rapid economic development, cardiovascular disease (CVD) has become the leading cause of death among Chinese adults. Physical inactivity and CVD risk factors such as obesity, hypertension, and dyslipidemia, are significantly related to the all-cause mortality in China [1]. Research suggests control of CVD risk factors and increased physical activity (PA) are two important strategies for reducing the burden of premature death among Chinese adults [1-3]. High levels of physical activity (PA) and cardiorespiratory fitness (CRF) are each associated with a favorable cardiovascular disease (CVD) risk profile. However, the relationship between CRF and obesity is still inconsistent across studies, and there has been no thorough exploration of the independent contribution of CRF to different CVD risk factors in Chinese women. This study investigated the relationship between CRF and CVD risk factors in 40-49 year old women.

\section{Methods}

Four hundred and sixty-nine women 40-49 years of age participated in a larger CVD surveillance study and completed an extensive questionnaire. Personal information included social background (age, sex, family income, and education), smoking, alcohol consumption, food frequency, previous disease history and family history. PA was assessed with the long Chinese version of the International Physical Activity Questionnaire (IPAQ) [5].

Two hundred and thirty-one women (age: $44.5 \pm 3.3 \mathrm{yr}$, BMI: $23.8 \pm 3.5 \mathrm{~kg} \cdot \mathrm{m}-2$ ) voluntarily agreed to undertake additional laboratory tests. Test contents and requirements were confirmed with all participants by telephone two days prior to visiting the lab. Each participant was free of any exercise testing contraindications [6]. Cycle ergometer exercise tests were conducted to assess CRF as indicated by maximal oxygen uptake (VO2max). Participants were categorized into three CRF levels (low, moderate and high). 


\section{Results}

Demographic characteristics and CVD risk factors are displayed in Tables 1 . The mean $\mathrm{VO}_{2 \max }$ was $27.35 \pm 5.53 \mathrm{ml} \cdot \mathrm{kg}^{-1} \mathrm{~min}^{-1}$, which was at a moderate level for the population's gender and age [6]. The average total weekly PA was $3476.29 \pm 3771.36 \mathrm{MET} \cdot \mathrm{min} \cdot$ week $^{-1}$, which was at the high level of PA according to the IPAQ scoring protocol (high level: total PA >3000 MET-minutes/week). A majority (72.3\%) of total weekly PA was comprised of non-leisure-time PA, including work-related PA (23.6\%), transport-related PA (29.7\%), and household-related PA (19.0\%).

References are cited in the text just by square brackets [1]. Two or more references at a time may be put in one set of brackets $[3,4]$. The references are to be numbered in the order in which they are cited in the text and are to be listed at the end of the contribution under a heading References, see our example below.

Table 1 Proportion of cardiovascular disease risk factors in 3 cardiorespiratory fitness groups

\begin{tabular}{|c|c|c|c|c|c|c|c|c|}
\hline \multirow[t]{2}{*}{ Variable $^{\mathrm{a}}$} & \multicolumn{8}{|c|}{ Fitness Level $^{\text {b }}$} \\
\hline & \multicolumn{2}{|c|}{ Low $(N=47)$} & \multicolumn{2}{|c|}{$\begin{array}{c}\text { Moderate }(\mathrm{N}= \\
\text { 92) }\end{array}$} & \multicolumn{2}{|c|}{ High ( $N=92)$} & \multicolumn{2}{|c|}{ Total $(\mathrm{N}=231)$} \\
\hline & \multicolumn{2}{|c|}{$\mathbf{N}(\%)^{\mathrm{c}}$} & \multicolumn{2}{|c|}{$\mathbf{N}(\%)$} & \multicolumn{2}{|c|}{ N (\%) } & \multicolumn{2}{|c|}{ N (\%) } \\
\hline Smoking & 2 & $(4.3 \%)$ & 4 & $(4.3 \%)$ & 6 & $(6.5 \%)$ & 12 & $(5.2 \%)$ \\
\hline Alcohol Drinking & 5 & $(10.6 \%)$ & 20 & $(21.4 \%)$ & 12 & $(12.5 \%)$ & 37 & $(16.0 \%)$ \\
\hline Overweight by body mass index & 17 & (36.5\%) & 27 & $(29.6 \%)$ & 23 & $(25.0 \%)$ & 67 & $(29.2 \%)$ \\
\hline Overweight by waist circumference & 12 & $(25.9 \%)$ & 25 & $(26.8 \%)$ & 15 & $(16.7 \%)$ & 52 & $(22.6 \%)$ \\
\hline Overweight by body fat \% & 30 & $(63.5 \%)$ & 34 & $(36.6 \%)$ & 31 & $(33.3 \%)$ & 95 & $(41.1 \%)^{d}$ \\
\hline Physical inactivity & 4 & $(8.2 \%)$ & 5 & $(5.6 \%)$ & 4 & $(4.2 \%)$ & 13 & $(5.6 \%)$ \\
\hline High fasting glucose & 3 & $(5.9 \%)$ & 9 & $(9.6 \%)$ & 10 & $(11.1 \%)$ & 22 & $(9.5 \%)$ \\
\hline High Total cholesterol & 9 & $(18.4 \%)$ & 20 & $(21.4 \%)$ & 23 & $(25.0 \%)$ & 52 & $(22.5 \%)$ \\
\hline Low HDL- cholesterol & 4 & $(7.9 \%)$ & 10 & $(10.7 \%)$ & 8 & $(8.3 \%)$ & 22 & $(9.5 \%)$ \\
\hline High LDL- cholesterol & 11 & $(23.7 \%)$ & 20 & $(21.4 \%)$ & 23 & $(25.0 \%)$ & 54 & $(23.3 \%)$ \\
\hline High systolic blood pressure & 2 & $(5.3 \%)$ & 9 & $(10.0 \%)$ & 4 & $(4.5 \%)$ & 15 & $(6.5 \%)$ \\
\hline High diastolic blood pressure & 4 & $(8.9 \%)$ & 9 & $(10.0 \%)$ & 4 & $(4.3 \%)$ & 17 & $(7.5 \%)$ \\
\hline ST-Depression during exercise & 3 & $(6.4 \%)$ & 5 & $(5.4 \%)$ & 3 & $(3.3 \%)$ & 11 & $(4.8 \%)^{d}$ \\
\hline Long QTC during exercise & 3 & $(6.4 \%)$ & 4 & $(4.3 \%)$ & 3 & $(3.3 \%)$ & 10 & $(4.3 \%)^{d}$ \\
\hline
\end{tabular}

${ }^{a}$ Reference value for abnormalities according to American College of Sports Medicine criteria.

${ }^{\mathbf{b}}$ According to $\mathrm{VO}_{2 \max }$, the least fit $20 \%$ were classified as low fit, the next $40 \%$ as moderately fit, and the top $40 \%$ as high fit.

${ }^{\mathbf{c}} \mathrm{N}$ denotes the number of participants with the CVD risk factor within each CRF group, \% indicates the percentage of participants with the CVD risk factor within each CRF group.

${ }^{d}$ Denotes significant difference $(P<0.01)$ in percentages among the three CRF groups.

Overall, CVD risk profiles of the moderate and high CRF groups were more favorable than that of the low CRF group. There were significant differences in total weekly PA among the three CRF groups $(\mathrm{P}<0.05)$, with total weekly PA being highest in the high CRF group and lowest in the low CRF group. Total weekly PA was significantly higher in the high CRF group than in the low CRF group (P $<0.05)$, with no significant difference between the moderate and low CRF group (P $>0.05)$. Higher CRF levels were also associated with significantly less BF\% $(\mathrm{P}<0.05)$ and lower $\mathrm{PWV}(\mathrm{P}<0.05)$, which indicated less stiffness of the vasculature. There were significant differences in BF\% between the moderate and low CRF group $(\mathrm{P}<0.05)$ and the high and low CRF group $(\mathrm{P}<0.05)$. PWV was significantly lower in the high CRF group than in the low CRF group $(\mathrm{P}<0.05)$. No significances were found in other variables $(\mathrm{P}>0.05)$.

The chi-square test revealed significant differences in the proportions of abnormal BF\%, ST segment depression, and long QTC interval across CRF groups $(\mathrm{P}<0.05)$ (Table 2). The proportion of participants with overweight status, ST segment depression, and long QTC interval during cycle 
exercise testing were lowest in the high CRF group and highest in the low CRF group. No significances existed for those variables across CRF groups $(\mathrm{P}>0.05)$.

After adjustment for a range of potential confounding factors including age, family history, income, education, smoking, diet, alcohol consumption and total PA, logistic regression indicated the odds ratio for having $\leqslant 1,2$, or $\geqslant 3$ CVD risk factors (Table 3). Odds ratios for having $\geqslant 3$ CVD risk factors in the low and moderate CRF groups were significantly higher compared with the high CRF group $(\mathrm{P}<0.05)$. The likelihood of having $\geqslant 3$ CVD risk factors in the low CRF group was 2.09 times greater than women in the high CRF group. Also, the low CRF group had significant less probability of having $\leqslant 1$ CVD risk factors $(\mathrm{P}<0.05)$. Table 3 also shows the that odds ratios of ST segment depression and long QTC interval during cycle exercise testing in the low and moderate CRF groups were significantly higher compared with the high CRF group $(\mathrm{P}<0.05)$. This indicated the probability of myocardial ischemia in the high CRF group was significantly less than in the moderate and low CRF groups.

\section{Discussion}

The purpose of the current study was to examine the association between objectively measured CRF and several CVD risk factors in middle-aged women. The results of the present study demonstrated that CRF was significantly inversely associated with CVD risk in this population with both moderate and high levels of CRF being associated with reduced risk. CRF is primarily a function of the heart's maximal ability to pump blood and the ability of skeletal muscle to extract and use oxygen. It is a more objective measure reflecting the PA level and cardiovascular function than self-reported PA which is prone to bias. CRF is influenced by both genetic and environmental factors. Environment, such as the frequency, intensity and duration of daily PA, has a significant effect on CRF, and appears to account for more of the variation in CRF than do genetic factors.

We observed that CRF was strongly and inversely associated with BF\%. As a more precise variable, BF\% measured by BIA indicated an association between fatness and CRF, while BMI failed to exclude the confounding effect of lean body mass. Regular PA has been shown to reduce the risks for obesity and metabolic disease at all ages. Recent studies [4] support the finding that higher CRF is related to lower risks of obesity for both men and women. For example, Irwin et al. [7] reported higher levels CRF predicted less body weight and lower BF\%. One study conducted in Hong Kong [8] also showed a significant inverse association between BF\% and CRF in women aged 55-94 years. Similar results from Wong et al. [8] demonstrated body fat mass and lean body mass were independently associated with VO2max in Chinese adults. The CRF level measured in our study was also consistent with results for adult women aged 40-49 years old who performed cycle exercise tests in these two studies [8,9]. CRF was also significantly negatively associated with PWV, which is a valid and reliable measurement of arterial stiffness. PWV indicates the general burden of atherosclerotic disease and subclinical damage from multiple risk factors over time, such as the effects of aging, smoking, and lipid metabolism [10]. In alignment with our results, recent studies have also discovered that CRF is associated with arterial stiffness. Boreham et al. [11] reported CRF was inversely related to PWV in young adults, and the relationship was independent of other CVD risk factors and daily PA levels. Gando et al. [12] reported higher CRF was associated with lower BP and arterial stiffness in older women. One possible reason for the relationship is that higher CRF minimizes age-related structural changes in the arterial wall related to an elevated overall content of elastin and a reduced calcium content. Another possibility is higher CRF may act to maintain endothelium-dependent vasodilation.

\section{Conclusion}

The total weekly PA of this population was high, and mainly consisted of non-leisure-time PA. Moderate and high levels of CRF measured by a cycle exercise test were significantly related to the 
CVD risk profile in 40-49 year old Chinese women. It seems reasonable to suggest a stronger emphasis be placed on increasing the CRF level of this population via regular PA, especially for those who are unfit. Further studies need to confirm the causal relationship between CRF and CVD risk for Chinese and Asian female populations.

\section{Acknowledgments}

This study was supported by the Fundamental Research Funds for the Central Universities (GK201603129) and MOE (Ministry of Education in China) Project of Humanities and Social Sciences (Project No.16XJC890001).

\section{References}

[1] He J, Gu D, Wu X, Reynolds K, Duan X, Yao C, Wang J, Chen C, Chen J, Wildman RP: Major causes of death among men and women in China. N Engl J Med 2005, 353(11):1124-1134.

[2] Gu D, He J, Duan X, Reynolds K, Wu X, Chen J, Huang G, Chen C, Whelton PK: Body weight and mortality among men and women in China. JAMA 2006, 295(7):776-783.

[3] Hu G, Pekkarinen H, Hanninen O, Yu Z, Guo Z, Tian H: Commuting, leisure-time physical activity, and cardiovascular risk factors in China. Med Sci Sports Exerc 2002, 34(2):234-238.

[4] Lee CD, Blair SN, Jackson AS: Cardiorespiratory fitness, body composition, and all-cause and cardiovascular disease mortality in men. Am J Clin Nutr 1999, 69(3):373-380.

[5] Hagstromer M, Oja P, Sjostrom M: The International Physical Activity Questionnaire (IPAQ): a study of concurrent and construct validity. Public Health Nutr 2006, 9(6):755-762.

[6] Thompson WR, Gordon NF, Pescatello LS: ACSM's guidelines for exercise testing and prescription. 8th edition. Edited by Thompson WR, Gordon NF, Pescatello LS. Philadelphia: Wolters Kluwer Lippincott Williams \& Wilkins; 2010:35-96.

[7] Irwin ML, Yasui Y, Ulrich CM, Bowen D, Rudolph RE, Schwartz RS, Yukawa M, Aiello E, Potter JD, McTiernan A: Effect of exercise on total and intra-abdominal body fat in postmenopausal women. JAMA 2003, 289(3):323-330.

[8] Yu R, Yau F, Ho S, Woo J: Cardiorespiratory fitness and its association with body composition and physical activity in Hong Kong Chinese women aged from 55 to 94 years. Maturitas 2011, 69(4):348-353.

[9] Wong SY, Chan FW, Lee CK, Li M, Yeung F, Lum CC, Choy DT, Woo J: Maximum oxygen uptake and body composition of healthy Hong Kong Chinese adult men and women aged 20-64 years. J Sports Sci 2008, 26(3):295-302.

[10] Cruickshank JK, Rezailashkajani M, Goudot G: Arterial Stiffness, Fatness, and Physical Fitness Ready for Intervention in Childhood and Across the Life Course? Hypertension 2009, 53(4):602-604.

[11] Boreham CA, Ferreira I, Twisk JW, Gallagher AM, Savage MJ, Murray LJ: Cardiorespiratory fitness, physical activity, and arterial stiffness: The Northern Ireland Young Hearts Project. Hypertension 2004, 44(5):721-726.

[12] Gando Y, Kawano H, Yamamoto K, Sanada K, Tanimoto M, Oh T, Ohmori Y, Miyatani M, Usui C, Takahashi E: Age and cardiorespiratory fitness are associated with arterial stiffening and left ventricular remodelling. J Hum Hypertens 2009, 24(3):197-206. 